\title{
ADAPTACIÓN Y RENDIMIENTO DE LA VARIEDAD DE FRIJOL INTA VAINA ROJA, PARA TOLERANCIA A SEQUÍA Y MANCHA ANGULAR EN CUATRO REGIONES DE NICARAGUA
}

\section{ADAPTATION AND PERFORMANCE OF THE BEAN VARIETY INTA VAINA ROJA FOR TOLERANCE TO DROUGHT AND LEAF SPOT DISEASE IN FOUR REGIONS OF NICARAGUA}

\author{
Molina Centeno Julio Cesar ${ }^{1}$, Cuadra Sergio ${ }^{1}$, Guzmán Mauricio ${ }^{1}$, Duarte Noel ${ }^{1}$, Llano Aurelio ${ }^{2}$ \\ ${ }^{1}$ Investigador zonal Granos Básicos, INTA. Email: molinacentenojuliocesar1@gmail.com \\ ${ }^{2}$ Investigador Nacional Frijol, INTA. Email; aureliollano@gmail.com
}

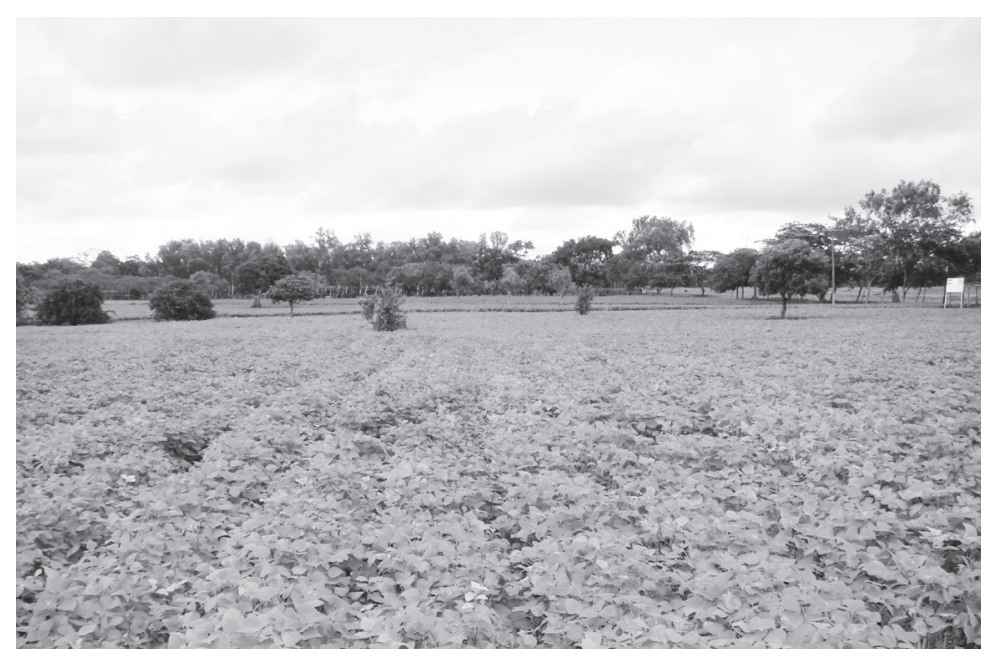

\section{RESUMEN}

El presente trabajo se llevó a cabo entre los años 2005 y 2007, con el objetivo de evaluar la adaptación y rendimiento de la variedad mejorada INTA Vaina Roja, en las zonas de Las Segovias, Centro Norte, Centro Sur y Pacífico Sur de Nicaragua. En el año 2006 se condujeron nueve ensayos de rendimiento, uno en Madriz, cinco en Estelí, uno en Matagalpa y dos en Jinotega. En las etapas preliminares de rendimiento, el ensayo incluyó 51 líneas F8, y en las pruebas avanzadas de rendimiento se incluyeron 34 líneas F9 y 14 líneas F8 y F9. Estos trabajos se realizaron de acuerdo a las condiciones agroecológicas de cada localidad bajo condiciones de secano. En las pruebas preliminares se utilizó una sola repetición, y en pruebas avanzadas, se utilizó un diseño de látice $6 \times 6$ y de bloques completos al azar con tres repeticiones. Los resultados obtenidos muestra que a nivel de experimentación las líneas 429 DFSZ 1509439 (2 $188 \mathrm{~kg} \mathrm{ha}^{-1}$ ), MIB 438 (2 $\left.644 \mathrm{~kg} \mathrm{ha}^{-1}\right), 426$ DFSZ 15094-39 (2 $182 \mathrm{~kg} \mathrm{ha}^{-1}$ ) e INTA Vaina Roja (2 $\left.013 \mathrm{~kg} \mathrm{ha}^{-1}\right)$ sobresalieron por su rendimiento de grano. Durante las evaluaciones, la variedad INTA Vaina Roja resultó tolerante a la enfermedad de mancha angular (Phaeoisariopsis griseola). Además, la variedad INTA Vaina Roja se validó en parcelas comerciales en campos de agricultores en 54 ambientes de los departamentos de Estelí, Madriz, Nueva Segovia, Matagalpa, Jinotega, Chontales y Carazo. En estas parcelas de validación, la variedad INTA Vaina Roja $\left(915 \mathrm{~kg} \mathrm{ha}^{-1}\right)$ superó en un $17 \%$ en los 54 sitios de validación a las variedades comerciales (784 $\mathrm{kg} \mathrm{ha}^{-1}$ ) utilizadas como testigos locales. Así mismo, dio los mejores rendimientos ( $\left.1343 \mathrm{~kg} \mathrm{ha}^{-1}\right)$ en 24 ambientes identificados como favorables con un $16 \%$ más que las variedades comerciales (1155
The present work was carried out between 2005 and 2007, with the aim of evaluating the adaptation and performance of the improved variety INTA Vaina Roja, in the regions of Las Segovias, North in the advanced yield studies 34 F9 lines and 14 F8 and F9 lines. This work was conducted according to agro-ecological conditions of each locality under dry land conditions. In the preliminary tests we used a single repetition, and in advanced tests, a lattice design $6 \times 6$ and randomized complete block with three replications was used. It was found that at experimental level 429 DFSZ 15094-39 (2 $\left.188 \mathrm{~kg} \mathrm{ha}^{-1}\right)$, MIB 438 (2 $\left.644 \mathrm{~kg} \mathrm{ha}^{-1}\right)$, 426 DFSZ 15094-39 (2 182 $\mathrm{kg} \mathrm{ha}^{-1}$ ) e INTA Vaina Roja (2 $\left.013 \mathrm{~kg} \mathrm{ha}^{-1}\right)$. Stood out for their grain yield. During the assessments, the INTA Vaina Roja variety was tolerant to leaf spot disease ( Phaeoisariopsis griseola). In addition, the INTA Vaina Roja variety was validated in commercial plots in farmers' fields in 54 environments of the departments of Estelí, Madriz, Nueva Segovia Matagalpa, Jinotega, Chontales and Carazo. In these test plots, yield form the variety INTA Vaina Roja $(915 \mathrm{~kg}$ $\mathrm{ha}^{-1}$ ) was higher by $17 \%$ in the 54 validation sites compared to commercial varieties $\left(784 \mathrm{~kg} \mathrm{ha}^{-1}\right)$ used as local control plot. It also gave the best yields ( $1343 \mathrm{~kg} \mathrm{ha}^{-1}$ ) in 24 environments identified as favorable yielding $16 \%$ more than commercial varieties (1 155 $\mathrm{kg} \mathrm{ha}^{-1}$ ) as well as in the 30 enviroments recognized as unfavorable the variety INTA Vaina Roja $\left(573 \mathrm{~kg} \mathrm{ha}^{-1}\right)$ had the highest yields , overtaking commercial varieties $\left(487 \mathrm{~kg} \mathrm{ha}^{-1}\right)$ by $18 \%$. In both situations the variety INTA Vaina Roja with coefficients of variation of 27.18 and $33.85 \%$ showed better stability than commercial varieties. During the process of experimentation and validation, 
kg ha- $\left.{ }^{1}\right)$. En los 30 ambientes identificados como desfavorables la variedad INTA Vaina Roja (573 $\mathrm{kg} \mathrm{ha}^{-1}$ ), tuvo los mayores rendimientos, superando a las variedades comerciales (487 $\left.\mathrm{kg} \mathrm{ha}^{-1}\right)$ en un $18 \%$. En ambas situaciones la variedad INTA Vaina Roja con coeficientes de variación de 27.18 y $33.85 \%$ presentó mejor estabilidad que las variedades comerciales. Durante el proceso de experimentación y de validación, se observó que la variedad INTA Vaina Roja presentó tolerancia a alta humedad en el suelo, lo que la hace ser un material ideal para adaptación al cambio climático. La variedad INTA Vaina Roja presentó una Tasa Marginal de Retorno de $991 \%$ superior a las variedades comerciales.

Palabras clave: mejoramiento vegetal, análisis de adaptabilidad, investigación en fincas.

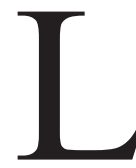

a importancia del cultivo de fríjol radica en que es la principal fuente de proteína vegetal en la dieta alimenticia de la población segoviana, pues posee alrededor de $22 \%$ de proteína. Es un generador de fuentes de ingresos para los productores, lo cual se demuestra con el área sembrada en la región de Las Segovias de aproximadamente 34363 hectáreas. Una de las limitantes en la producción de este cultivo es la alta incidencia de sequía, mosaico dorado y mancha angular en la zona seca de esta región, lo cual reduce los rendimientos de grano hasta en unos $700 \mathrm{~kg} \mathrm{ha}^{-1}$.

El INTA en colaboración con el Centro Internacional de Agricultura Tropical (CIAT), han desarrollado una línea de trabajo orientada a generar genotipos de fríjol para tolerancia a sequía y mancha angular. En este sentido, en las épocas de primera y postrera del ciclo agrícola 2006-2007, fue evaluado un vivero para alto contenido de hierro en el departamento de Estelí. Como resultado de este trabajo, se seleccionaron los mejores 13 materiales por su potencial de rendimiento, color de grano y tolerancia a sequía y mancha angular, los que fueron evaluados en el ciclo 2007 - 2008 en tres localidades del departamento de Estelí, y una localidad del departamento de Jinotega, permitiendo la identificación de los genotipos 429 DFSZ 1504-39 (2 $\left.188 \mathrm{~kg} \mathrm{ha}^{-1}\right), 516$ DFBS 15089-22 (2 $\left.130 \mathrm{~kg} \mathrm{ha}^{-1}\right), 426$ DFSZ 15094-39 (2 $\left.182 \mathrm{~kg} \mathrm{ha}^{-1}\right), 703 \mathrm{SM}$ $15216-11$ (2 $\left.098 \mathrm{~kg} \mathrm{ha}^{-1}\right)$, MIB 438 (2 $\left.644 \mathrm{~kg} \mathrm{ha}^{-1}\right), 618 \mathrm{DFSZ}$ 15132-12 (2 $\left.012 \mathrm{~kg} \mathrm{ha}^{-1}\right)$, INTA Vaina Roja (2 $013 \mathrm{~kg} \mathrm{ha}^{-1}$ ), MIB 451 (2 $017 \mathrm{~kg} \mathrm{ha}^{-1}$ ) y SER 82 (2 $\left.044 \mathrm{~kg} \mathrm{ha}^{-1}\right)$, que dieron rendimientos superiores a la variedad comercial INTA Rojo (2011 kg ha-1). De este grupo, se destacaron por su mayor precocidad y color de grano los materiales INTA Vaina Roja y SER 82. Estas variedades experimentales fueron validadas en los ciclos agrícolas 2008-2009 y 2009-2010 en 54 localidades de los departamentos de Estelí, Madriz y Nueva Segovia. En todos estos trabajos la línea INTA VAINA ROJA mostró buena adaptación y estabilidad del rendimiento de grano tanto en ambientes favorables como desfavorables (Molina et al., 2 010). El objetivo del presente estudio fue we observed that the INTA Vaina Roja variety showed tolerance to high humidity condition in the soil, making it an ideal material for the adaptation to climate change. The INTA Vaina Roja variety presented a marginal rate of return of $991 \%$ superior to commercial varieties.

determinar la adaptación y estabilidad del rendimiento de la variedad de fríjol INTA Vaina Roja en cuatro regiones de Nicaragua.

\section{MATERIALES Y MÉTODOS}

Evaluación del rendimiento de la línea INTA Vaina Roja en la fase de experimentación. Durante la época de primera del ciclo agrícola 2006-2007, se condujeron tres viveros; uno en Madriz, uno en Estelí y uno en Matagalpa. En los viveros, entre los 51 genotipos F8 se incluyó la línea INTA Vaina Roja y el testigo INTA Rojo, que es la principal variedad recomendada para las zonas frijoleras de Nicaragua. En estos trabajos se utilizó una sola repetición, y parcelas de un surco de cinco metros de largo. También, en la época de primera y postrera del período 2006-2007, se establecieron cuatro ensayos de líneas avanzadas F8 y F9 en los municipios de Condega, La Concordia y La Trinidad. En estos ensayos se utilizó un diseño de Bloques Completos al Azar con tres repeticiones y parcelas de cuatro surcos de cinco metros de longitud. Además, en la época de postrera del 2006, se estableció un trabajo sobre la evaluación de 34 líneas F9 de frijol en los municipios de Condega y La Concordia, bajo un diseño de látice $6 \times 6$ con parcelas experimentales de un surco de cinco metros de largo. La fecha de siembra de los experimentos fue en los meses de junio y septiembre. Los ensayos se condujeron siguiendo las recomendaciones agronómicas del cultivo.

En todas las localidades se tomaron datos de reacción a enfermedades que ocurrieron durante el ciclo, la fenología de las variedades y el rendimiento. El rendimiento de grano se calculó en $\mathrm{kg} \mathrm{ha}^{-1}$ al 14\% de humedad. Los datos de rendimiento se sometieron a un análisis de varianza individual y después se realizó un análisis combinado en los casos que se tenía más de un experimento. Para comparar las medias de tratamiento se utilizó la prueba de Tukey a un nivel de significancia del $0.05 \%$.

Evaluación de reacción a enfermedades. Durante la conducción de los ensayos, se calificó la reacción de los 
genotipos evaluados a la incidencia del virus del mosaico dorado y de mancha angular en la localidad de El CulceCondega, con una escala de 1 a 9 (Shoonhoven y Pastor Corrales, 1987), cuyos valores son: 1 a $3=$ resistente, 4 a $6=$ intermedio y 7 a $9=$ susceptible. Esta medición se realizó en la etapa reproductiva del cultivo.

Evaluación de la línea INTA Vaina Roja en parcelas de validación. La línea INTA Vaina Roja Se evaluó en parcelas comerciales en 54 ambientes de cuatro regiones de Nicaragua, durante el ciclo agrícola 2007-2008, y se comparó con variedades comerciales utilizadas en cada región (mejoradas, acriolladas y criollas). Las siembras se realizaron al tiempo que se establecieron los lotes comerciales en cada localidad durante los meses de junio, septiembre y noviembre. La superficie por lote de validación fue variable en cada sitio de acuerdo a la disponibilidad de semilla (350 a $500 \mathrm{~m}^{2}$ ). El rendimiento se calculó en $\mathrm{kg} \mathrm{ha}^{-1}$ en base al área total sembrada, y en aquellos casos en que hubo fallas en la parcela, se hicieron cuatro muestreos de cinco $\mathrm{m}^{2}$ por variedad. Estas parcelas de validación se establecieron en terrenos de agricultores cooperantes, los que se encargaron de la conducción agronómica y cosecha de las mismas. El rendimiento obtenido al $14 \%$ de humedad en cada localidad, se consideró como un ambiente. Con los datos recopilados se realizó un análisis haciendo uso del análisis de estabilidad modificada propuesto por Hildebrand y Poey (1989) a través del programa de Excel. El rendimiento de grano obtenido por la variedad experimental INTA Vaina Roja y las variedades utilizadas como testigo local, se relacionó con el índice ambiental (rendimiento promedio de ambos materiales en cada localidad), mediante el modelo de regresión simple, basada en el siguiente modelo:

$\mathrm{Yi}=\mathrm{a}+\mathrm{be}$

Donde:

Yi $=$ Rendimiento del i-ésimo tratamiento

$\mathrm{E}=$ índice ambiental
Tabla 1. Ubicación de áreas de validación con la variedad experimental INTA Vaina Roja en 54 localidades de Nicaragua, 2007

\begin{tabular}{|c|c|c|c|}
\hline Departamentos & Municipio & Localidad & Testigo local \\
\hline \multirow{13}{*}{ Estelí } & Condega & El Espino & Estelí 150 \\
\hline & Pueblo Nuevo & Los Horcones & Luisito \\
\hline & La Trinidad & Tomabú Arriba & Rojo de Seda \\
\hline & La Trinidad & Tamabú Abajo & Rojo de Seda Mejorado \\
\hline & La Trinidad & Potrerillo & Chile Rojo \\
\hline & La Trinidad & Tomabú postrera & Rojo de Seda \\
\hline & Estelí & El Espinal 1 & Cuarentano \\
\hline & Estelí & El Espinal 2 & INTA Rojo \\
\hline & La Trinidad & Las Limas & Cuarentano \\
\hline & San Nicolás & Las Puertas & Frijol Negro \\
\hline & Condega & El Culce & INTA Rojo \\
\hline & Estelí & San Antonio & Río Rojo \\
\hline & Pueblo Nuevo & Cofradía & JM Pueblo Nuevo \\
\hline \multirow{5}{*}{ Madriz } & San Lucas & La Manzana & Zamorano \\
\hline & San Lucas & Moropoto & INTA Rojo \\
\hline & San Lucas & Unile & JM Pueblo Nuevo \\
\hline & Palacaguina & La Concepción & INTA Rojo \\
\hline & Totogalpa & Quebrada Grande & Chile Rojo \\
\hline \multirow{5}{*}{ Matagalpa } & Sebaco & El Salto 1 & INTA Rojo \\
\hline & Sebaco & El Salto 2 & INTA Rojo \\
\hline & Matagalpa & Nuestra Tierra & DOR 364 \\
\hline & San Ramón & La Granja 1 & Estelí 90 \\
\hline & San Ramón & La Granja 1.1 & Estelí 90 \\
\hline \multirow{9}{*}{ Jinotega } & Jinotega & Chagüite Grande 1 & INTA Masatepe \\
\hline & Jinotega & Las Pilas 1 & Waliceño \\
\hline & Jinotega & Chagüite Grande 2 & INTA Masatepe \\
\hline & La Concordia & El Ocote & Estelí 150 \\
\hline & Jinotega & Chagüite Grande 2 & INTA Canela \\
\hline & Jinotega & Chagüite Grande 1.1 & INTA Canela \\
\hline & Jinotega & Los Robles & SRC 2-18-1 \\
\hline & Jinotega & Las Pilas 1.1 & INTA Canela \\
\hline & Jinotega & Las Pilas 2 & INTA Canela \\
\hline \multirow{13}{*}{ Carazo } & La Conquista & La Vainilla 1 & INTA Precoz \\
\hline & Diriamba & Zacate Verde & INTA Precoz \\
\hline & La Conquista & Santa Gertrudis & INTA Precoz \\
\hline & Diriamba & El Aguacate 2 & INTA Precoz \\
\hline & La Conquista & Buena Vista 1 & INTA Precoz \\
\hline & Diriamba & El Aguacate 2.1 & INTA Precoz \\
\hline & Diriamba & Las Mercedes & INTA Precoz \\
\hline & La Conquista & La Vainilla 1.1 & INTA Precoz \\
\hline & La Conquista & La Vainilla 1.2 & INTA Precoz \\
\hline & La Conquista & Buena Vista 1.1 & INTA Precoz \\
\hline & Diriamba & El Carrizal & INTA Precoz \\
\hline & Dolores & Lomas de Gavilán & INTA Precoz \\
\hline & Nandaime & Nandarola & INTA Precoz \\
\hline \multirow{9}{*}{ Chontales } & El Rama & Kisilaya & Rojo Nacional \\
\hline & Nueva Guinea & Montevideo & Rojo Nacional \\
\hline & Nueva Guinea & Río Plata & Chile Tico \\
\hline & El Rama & Mataka & Rojo Nacional \\
\hline & Nueva Guinea & Nueva Guinea & Chile Tico \\
\hline & $\begin{array}{l}\text { Muelle de los } \\
\text { Bueyes }\end{array}$ & Las Pavas & Rojo Nacional \\
\hline & Los Santos & Los Santos & Chile Tico \\
\hline & Nueva Guinea & Gerusalen & Chile Tico \\
\hline & El Rama & El Areno & Rojo Nacional \\
\hline
\end{tabular}


Estudios de la calidad del grano. La calidad del grano, se valoró con cada uno de los productores cooperantes después de la cosecha de las parcelas. Para ello, se orientó que la esposa de cada productor cocinara la misma cantidad de grano de la línea INTA Vaina Roja y de las variedades comerciales utilizadas como testigos locales, y que lo dejara para el siguiente día. Después se les preguntó que si la variedad INTA Vaina Roja cumplía con los criterios establecidos por ellos.

\section{RESULTADOS Y DISCUSIÓN}

Evaluación del rendimiento de grano de la línea INTA Vaina Roja en la fase de experimentación. A nivel de pruebas preliminares de rendimiento (vivero de 51 líneas F8), se identificaron 34 líneas promisorias con base a su potencial productivo, arquitectura de planta y contenido de hierro. En este grupo sobresalieron 11 materiales por su potencial de rendimiento y color de grano, dentro de los cuales se incluyó la línea INTA Vaina Roja. En el caso de la evaluación de 34 líneas promisorias realizada en los municipios de Condega y La Concordia en el año 2006, se encontró que 12 genotipos mostraron buenos rendimientos de grano con respecto al testigo local INTA Rojo (tabla 2), dentro de los cuales se encuentra INTA Vaina Roja. En los cuatro trabajos efectuados como pruebas avanzadas de rendimiento realizadas en los municipios de Condega (2), La Trinidad (1) y La Concordia (1) en las épocas de primera y postrera del 2006, las líneas 429 DFSZ 15094-39 (2 $\left.188 \mathrm{~kg} \mathrm{ha}^{-1}\right)$, MIB 438 (2 $644 \mathrm{~kg} \mathrm{ha}^{-}$ $\left.{ }^{1}\right), 426$ DFSZ 15094-39 (2 $\left.182 \mathrm{~kg} \mathrm{ha}^{-1}\right)$ e INTA Vaina Roja (2 $013 \mathrm{~kg} \mathrm{ha}^{-1}$ ), dieron los más altos rendimientos de grano, de las cuales se destaca la línea INTA Vaina Roja por su mejor color de grano (tabla 2).

Tabla 2. Rendimiento obtenido en $\mathrm{kg} \mathrm{ha}^{-1}$ con la variedad INTA Vaina Roja a nivel experimental, 2006

\begin{tabular}{|c|c|c|c|c|}
\hline Actividad & Municipio & Línea & $\begin{array}{l}\text { Rendimiento } \\
\qquad\left(\mathrm{kg} \mathrm{ha}^{-1}\right)\end{array}$ & $\begin{array}{c}\text { Color de } \\
\text { grano }\end{array}$ \\
\hline \multirow{12}{*}{$\begin{array}{l}\text { Evaluación de vivero } \\
\text { de } 51 \text { líneas F8 de } \\
\text { frijol rojo, para alto } \\
\text { contenido de hierro }\end{array}$} & \multirow{12}{*}{$\begin{array}{l}\text { San Lucas, } \\
\text { Condega } \\
\text { y Matagalpa } \\
\text { (Primera 2006) }\end{array}$} & 427 DFSZ 15094-39-1 & 1634 & 5 \\
\hline & & 428 DFSZ 15094-39-4 & 1741 & 2 \\
\hline & & 516 DFBS $15089-22-1$ & 1596 & 3 \\
\hline & & 519 DFBS 15089-22-1 & 1652 & 2 \\
\hline & & 578 DFSZ 15094-43-3 & 1654 & 5 \\
\hline & & 599 NFSZ 15171-09-2 & 1575 & 5 \\
\hline & & 618 BFSZ 15132-12-5 & 1738 & 3 \\
\hline & & INTA Vaina Roja & 1529 & 2 \\
\hline & & 628 SM 15212-33-4 & 1764 & 2 \\
\hline & & 703 SM 15216-11-3 & 1660 & 3 \\
\hline & & 703 SM 15216-11-5 & 1609 & 3 \\
\hline & & INTA Rojo & 1507 & 5 \\
\hline Evaluación de 34 líneas & Condega & 428 DFSZ 15094-39-1 & 792 & 5 \\
\hline F9 de frijol rojo, para & y La Concordia & 429 DFSZ 15094-39-4 & 797 & 4 \\
\hline alto contenido de hierro & (Postrera 2006) & 523 DFBS 15092-04-3 & 706 & 4 \\
\hline
\end{tabular}

Evaluación de reacción a enfermedades. La enfermedad de la mancha angular (Phaeoisariopsis griseola) se evaluó en la localidad de El Culce - Condega en el ciclo agrícola 2006-2007. Las variedades que mostraron mayor tolerancia a mancha angular fueron: MIB 438 (3.33) e INTA Vaina Roja (3.00), y las más susceptibles resultaron ser los materiales SER 155 (7.00), SER 82 (7.00) y la variedad comercial INTA Rojo (7.67), (tabla 3).

Tabla 3. Reacción a Mancha angular, días a flor y aspecto del grano de 14 líneas de frijol rojo, El Culce 2006

\begin{tabular}{lccc}
\hline Genotipo & Días a Flor & Mancha angular & Color de grano \\
\hline 518 DFBS 15089-22 & 36 & 5.67 & 4.0 \\
427 DFSZ 15094-39 & 36 & 5.00 & 4.67 \\
429 DFSZ 15094-39 & 36 & 5.33 & 4.83 \\
516 DFBS 15089-22 & 36 & 5.00 & 4.17 \\
426 DFSZ 15094-39 & 36 & 6.33 & 4.33 \\
SER 199 & 36 & 5.67 & 4.50 \\
703 SM 15216-11 & 35 & 6.00 & 3.67 \\
617 DFSZ 15132-12 & 33 & 6.00 & 5.00 \\
MIB 438 & 35 & 3.33 & 3.83 \\
618 DFSZ 15132-12 & 34 & 5.33 & 4.17 \\
INTA Vaina Roja & 33 & 3.0 & 2.00 \\
MIB 451 & 33 & 5.33 & 4.33 \\
SER 155 & 32 & 7.00 & 3.5 \\
SER 82 & 32 & 7.00 & 2.5 \\
INTA Rojo (T.L.) & 34 & 7.67 & 4.50
\end{tabular}

Medias con la misma letra no difieren estadísticamente, según Tukey $\mathrm{p}(0.05)$.

Evaluación de la variedad INTA Vaina Roja en parcelas de validación. Las condiciones agro climáticas ocurridas durante los ciclos agrícolas de evaluación (datos no disponibles) fueron en la mayoría de los casos desfavorables al cultivo por las condiciones de sequía que se presentaron durante el ciclo del cultivo. A nivel de las 54 localidades, se encontró que la variedad INTA Vaina Roja $\left(915 \mathrm{~kg} \mathrm{ha}^{-1}\right)$ superó a las variedades comerciales utilizadas como testigo local (784 $\mathrm{kg} \mathrm{ha}^{-1}$ ) en un $17 \%$. El comportamiento de las variedades está definido por el promedio del rendimiento obtenido en todas las localidades, el coeficiente de variación y la $\mathrm{R}^{2}(\%)$. Este valor de la $\mathrm{R}^{2}$ nos da una idea, de 
que los resultados obtenidos están relacionados con el comportamiento intrínsico del tratamiento o debido a factores externos. En la tabla 4, se observa que la línea INTA Vaina Roja, obtuvo el más alto valor de $\mathrm{R}^{2}$ de $95 \%$. Este valor alto de la $\mathrm{R}^{2}$ explica que estos resultados están influenciados por la parte genética de la variedad. Además, se encontró que el material INTA Vaina Roja tuvo el valor más bajo de coeficiente de variación (52.13\%) en comparación a las variedades comerciales $(58.03 \%)$, lo que demuestra que tiene mayor estabilidad a través de los ambientes evaluados.

Tabla 4. Rendimiento en $\mathrm{kg} \mathrm{ha}^{-1}$, coeficiente de regresión obtenido con la variedad INTA Vaina Roja y variedades comerciales en 54 localidades de cuatro regiones de Nicaragua, 2,007

\begin{tabular}{lcc}
\hline \multirow{2}{*}{ Localidad } & \multicolumn{2}{c}{ Variedades } \\
& INTA Vaina Roja & Variedades comerciales \\
\hline Media & 915 & 784 \\
CV (\%) & 52.13 & 58.03 \\
R$\left.^{2} \%\right)$ & 0.95 & 0.92 \\
Desv. Estándar & 477 & 455 \\
Mínimo & 184 & 129 \\
Máximo & 2208 & 250 \\
\% sobre el testigo & 117 & 100 \\
\hline
\end{tabular}

Cuando los rendimientos obtenidos en una localidad son superiores al promedio general, se considera el ambiente como bueno. El análisis de estabilidad modificada permitió la identificación de dos dominios de recomendación "pobres y buenos". 24 ambientes fueron identificados como buenos, en donde la variedad INTA Vaina Roja (1 $343 \mathrm{~kg} \mathrm{ha}^{-1}$ ) produjo rendimientos superiores a las variedades comerciales (1 155 $\mathrm{kg} \mathrm{ha}^{-1}$ ), superándolas en un $16 \%$, y coeficiente de variación de $27.18 \%$, considerado de mejor estabilidad que las variedades comerciales que alcanzaron un $36.71 \%$ (tabla 5).

Tabla 5. Rendimiento promedio de grano, coeficientes de regresión y de variación para 24 ambientes buenos, donde el índice ambiental fue mayor de $850 \mathrm{~kg} \mathrm{ha}^{-1}$

\begin{tabular}{lcc}
\hline Localidad & \multicolumn{2}{c}{ Variedades } \\
& INTA Vaina Roja & Testigos locales \\
\hline Media & 1343 & 1155 \\
CV $(\%)$ & 27.18 & 36.71 \\
$\%$ sobre el testigo & 116 & 100 \\
\hline
\end{tabular}

Como ambientes pobres se consideran aquellos que producen rendimientos por debajo del promedio general. En este estudio se encontraron 30 ambientes de este tipo (tabla 6), lo cual se debe principalmente a la sequía incidente y al mal manejo agronómico que se le dio al cultivo en algunos casos. Bajo las condiciones de clima y manejo al cultivo que se dieron en estas 30 localidades, la variedad INTA Vaina Roja (573 $\mathrm{kg} \mathrm{ha}^{-1}$ ) superó en un 18\% a las variedades utilizadas como testigos locales (487 $\mathrm{kg} \mathrm{ha}^{-1}$ ). En cuanto a la estabilidad de estos materiales, la variedad INTA Vaina Roja presentó valores de coeficientes de variación (33.85\%) más bajos que los obtenidos por las variedades comerciales (36.14\%). Estos valores son considerados como aceptables, lo que demuestra que este genotipo tiene una buena estabilidad del rendimiento de grano en este tipo de ambientes.

Tabla 6. Rendimiento promedio de grano, coeficientes de variación y regresión para 30 ambientes pobres donde el índice ambiental fue menor de $850 \mathrm{~kg} \mathrm{ha}^{-1}$

\begin{tabular}{lcc}
\hline Localidad & \multicolumn{2}{c}{ Variedades } \\
& INTA Vaina Roja & Testigos Locales \\
\hline Media & 573 & 487 \\
CV $(\%)$ & 33.85 & 36.14 \\
$\%$ sobre el testigo & 118 & 100 \\
\hline
\end{tabular}

En el gráfico de estabilidad, se observa que la variedad INTA Vaina Roja tuvo una buena estabilidad a través de los ambientes, con un mejor comportamiento en ambientes pobres y buenos con respecto a las variedades comerciales.

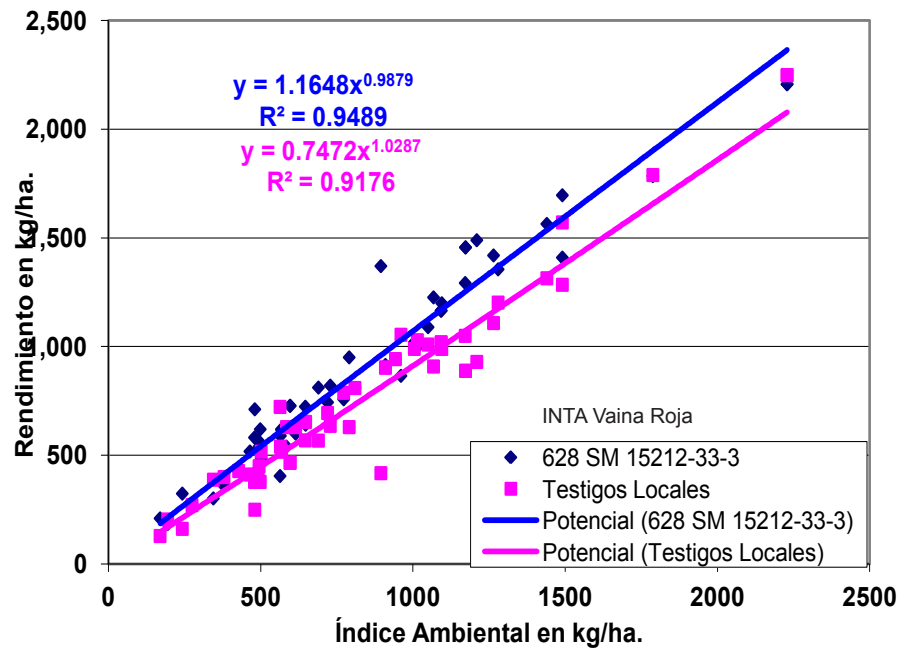

Figura 1. Dispersión y línea de tendencia de las variedades de frijol INTA Vaina Roja y testigos locales, en 54 localidades de Nicaragua, 2007.

Análisis económico. El propósito de hacer un análisis económico es el de obtener suficiente evidencia de que las opciones tecnológicas que se están proponiendo son factibles económicamente para los productores usuarios, en términos de generación de beneficios directos e indirectos medidos por unidades monetarias.

Por la razón antes expuesta se procedió a realizar el análisis económico basado en la teoría del presupuesto parcial propuesto por el CIMMYT, para conocer con exactitud los beneficios netos y la tasa de retorno marginal que obtendrá el productor al adoptar la alternativa tecnológica propuesta. 
Costos que varían. Los costos que varían son los costos (por hectárea) relacionados con los insumos comprados, mano de obra y la maquinaria, que varía de un tratamiento a otro. Los costos que varían en este caso es el precio de la semilla, debido a que las demás actividades relacionadas con el manejo agronómico del cultivo, son similares. Los costos de la semilla de las líneas propuestas (INTA Vaina Roja $=\mathrm{C} \$$ 1200 ) son $25 \%$ más alto con respecto al testigo (C\$900), siendo esta la semilla de la cosecha anterior que utiliza el productor en sus parcelas.

Análisis de presupuesto parcial. Se analizaron los datos con base en la tecnología de evaluación económica propuesta por el CYMMYT en 1988, para este fin se analizó el presupuesto parcial donde se trabajó un tratamiento alternativo (INTA Vaina Roja Vrs el testigo local). Se presentan los rendimientos obtenidos en todo el sitio del dominio de recomendación para cada tratamiento en base a los rendimientos medios. Para ajustar los rendimientos se redujo el 5\% por mal manejo de postcosecha.

El precio del kilogramo a nivel comercial durante la época de postrera del año 2007 se estimó por C\$26.4 el kilogramo. El precio de los frijoles, presenta una variación cíclica con el pasar de las épocas, pero aumenta de manera progresiva hasta alcanzar precios más altos en la época de menores ofertas, cuando el producto está escaso en el mercado.

Como se puede observar los beneficios netos de la tecnología propuesta fueron superiores a los del testigo, siendo los costos que varían más altos los de la línea propuesta, sin embargo los rendimientos vienen a compensar estos costos y por ende las utilidades netas.

Tabla 7. Análisis de presupuesto Parcial

\begin{tabular}{lcr}
\hline Actividad & INTA Vaina Roja & Testigo \\
\hline Rendimiento medio $\left(\mathrm{kg} \mathrm{ha}^{-1}\right)$ & 915.0 & 784.0 \\
Rendimiento ajustado al 5\% $\left(\mathrm{kg} \mathrm{ha}^{-1}\right)$ & 869.0 & 745.0 \\
Precio de venta $\mathrm{kg}$ & 26.4 & 26.4 \\
Beneficio bruto de campo $\left(\mathrm{C} \$ \mathrm{ha}^{-1}\right)$ & 22942.0 & 19668.0 \\
Costos que varían (semilla) & 1200.0 & 900.0 \\
Beneficios netos & 21742.0 & 18768.0 \\
\hline
\end{tabular}

Análisis de Dominancia. El tratamiento testigo (semilla de variedades comerciales); fue el que presentó menores costos variables (precio de la semilla). Con este tratamiento se obtuvieron menores rendimientos de grano y beneficios netos comparados con la tecnología propuesta. El análisis de dominancia se efectuó ordenando los tratamientos de menores a mayores costos totales que varían. Según la metodología del CIMMYT (1988), un tratamiento es dominado cuando tiene beneficios netos menores o iguales y costos variables mayores que cualquier otro tratamiento. El análisis de dominancia permite concluir que el testigo local, a pesar de presentar menores costos, fue dominado.

Análisis marginal. Con base al presupuesto parcial, se efectuó al análisis marginal para determinar la variación porcentual de costos y beneficios obteniendo como resultado una tasa marginal de retorno para la variedad INTA Vaina Roja, de $991 \%$, lo que significa que si los productores aceptan el cambio tecnológico, los ingresos por cada córdoba invertido por hectárea en la variedad INTA Vaina Roja, serían de 9.91 córdobas adicionales (tabla 8). Esta situación sugiere que la tecnología propuesta INTA Vaina Roja es rentable económicamente.

Estos resultados económicos tienen relación con la opinión de los productores sobre las características favorables (mayor rendimiento de grano, buena arquitectura de planta y resistencia a mancha angular) en relación a las variedades mejoradas comerciales utilizadas por los productores.

\begin{tabular}{lccccc}
\hline Tecnologías & $\begin{array}{c}\text { Total costos } \\
\text { que varían }\end{array}$ & $\begin{array}{c}\text { Costo } \\
\text { marginal }\end{array}$ & $\begin{array}{c}\text { Beneficios } \\
\text { netos }\end{array}$ & $\begin{array}{c}\text { Beneficio } \\
\text { marginal } \\
(\mathrm{C} \text { /ha })\end{array}$ & TRM (\%) \\
\hline Testigo & 900 & 300 & 18768 & 2974 & 99.1 \\
INTA Vaina Roja & 1200 & & 21742 & &
\end{tabular}

Estudio de la calidad del grano. En la mayoría de los casos las amas de casa que colaboraron con las parcelas de validación, manifestaron que la línea INTA Vaina Roja tiene un sabor aceptable del caldo, y una buena consistencia del grano, aun dejándolo de un día a otro.

Los comerciantes opinan que INTA Vaina Roja posee buenas caracteristicas comerciales, por su parte, los consumidores la aceptan por sus propiedades culinarias como la consistencia del grano cocido, tiempo aceptable de cocción y caldo ralo, similar a las veriedades criollas.

\section{CONCLUSIONES}

A nivel experimental fueron identificadas las líneas 429 DFSZ 15094-39 (2 $\left.188 \mathrm{~kg} \mathrm{ha}^{-1}\right)$, MIB 438 (2 $\left.644 \mathrm{~kg} \mathrm{ha}^{-1}\right), 426$ DFSZ 15094-39 (2 $\left.182 \mathrm{~kg} \mathrm{ha}^{-1}\right)$ e INTA Vaina Roja (2 $\left.013 \mathrm{~kg} \mathrm{ha}^{-1}\right)$, por su buen potencial de rendimiento y tolerancia a sequía, de las cuales se destaca por el color de grano y tolerancia a mancha angular, la línea INTA Vaina Roja.

En las 54 localidades de estudio, la variedad INTA Vaina Roja (915 kg ha-1) superó a los testigos locales (784 $\mathrm{kg} \mathrm{ha}^{-1}$ ) en un $17 \%$. Además se encontró que este genotipo presentó una mejor estabilidad del rendimiento de grano y se adapta bien a las diferentes prácticas que realizan los productores al cultivo de frijol, ya que tuvo los valores más 
bajos de coeficientes de variación (52.13\%) y los más altos de $\mathrm{R}^{2}$ (0.95).

Para los 24 ambientes considerados como buenos la variedad INTA Vaina Roja (1 $343 \mathrm{~kg} \mathrm{ha}^{-1}$ ) produjo los mayores rendimientos de grano con valores de $16 \%$ por encima de las variedades comerciales (1 $\left.155 \mathrm{~kg} \mathrm{ha}^{-1}\right)$. También, para los 30 ambientes considerados como malos, la variedad INTA Vaina Roja $\left(573 \mathrm{~kg} \mathrm{ha}^{-1}\right)$ superó en un $18 \%$ a las variedades utilizadas como testigos locales $\left(487 \mathrm{~kg} \mathrm{ha}^{-1}\right)$. En ambas situaciones la variedad INTA Vaina Roja con coeficientes de variación de 27.18 y $33.85 \%$ presentó mejor estabilidad que las variedades comerciales.

La variedad INTA Vaina Roja presentó una Tasa Marginal de Retorno de 991\%.

Es preciso difundir y registrar la variedad INTA Vaina Roja, como una nueva variedad comercial en Nicaragua.

\section{REFERENCIAS BIBLIOGRÁFICAS}

Beebe, S. 2,005. Mejorando Cultivos para una Mejor Nutrición. HarvestPlus. CIAT, Cali, Colombia. CIMMYT, 1988. La formulación de recomendaciones a partir de datos agronómicos; un manual metodológico de evaluación económica. MX, D.F. 79 p.

Fusell, LK; Bidinger, FR; Bieler, P. 1991. Crop physiology and breeding for drought tolerance: research and development. Field Crops Res. 27:183-199.

Hildebrand, PE; Poey, F. 1989. Ensayos agronómicos en fincas. Según el enfoque de sistemas agropecuarios. Editorial Agropecuaria Latinoamericana. USA. 134 p.

Kohashi-Shibata, J. 1990. Morfología y fisiología del frijol (Phaseolus vulgaris L.). Tierra 8:3-17.

Ludlow, MM; Muchow, RC. 1990. A critical evaluation of trails for improving Crop yields in water-limited environments. Adv. Agron. 43:107-153.

Molina, J; Valdivia, R. 2006. Evaluación de 14 líneas F8 de frijol rojo para alto contenido de hierro. En: Informe Técnico, Proyecto de Investigación y Desarrollo, INTA Las Segovias. Estelí, NI.

Radulovich, R; Karremans, J. 1993. Validación de tecnologías en sistemas agrícolas. Centro Internacional de Agricultura Tropical. Costa Rica. 95 p.

Tosquy, O; Sierra, M; Rodríguez, F; Castillo, O; Tinoco, A; Sandoval, A; Uribe, S. 1,995. Validación del híbrido de maíz de cruza doble H-512 en el estado de Veracruz. México. Agronomía Mesoamericana 6:93-97.

Villee, C. 1,990. Biología. MX. MC Grawn Hill. 